\title{
Vers une édition électronique de la glose d'Oxford
}

Towards an Electronic Edition of the Oxford Gloss

\section{Emmanuelle Kuhry}

\section{(2) OpenEdition}

Journals

Édition électronique

URL : https://journals.openedition.org/revuehn/450

DOI : 10.4000/revuehn.450

ISSN : 2736-2337

Éditeur

Humanistica

\section{Référence électronique}

Emmanuelle Kuhry, «Vers une édition électronique de la glose d'Oxford », Humanités numériques [En ligne], 2 | 2020, mis en ligne le 01 juin 2020, consulté le 18 juillet 2021. URL : http:// journals.openedition.org/revuehn/450; DOI : https://doi.org/10.4000/revuehn.450

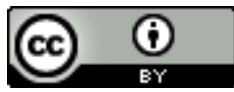

Les contenus de la revue Humanités numériques sont mis à disposition selon les termes de la Licence Creative Commons Attribution 4.0 International. 


\title{
humanités \\ numériques
}

\section{Vers une édition électronique de la glose d'Oxford}

\section{Towards an Electronic Edition of the Oxford Gloss}

\author{
Emmanuelle Kuhry
}

\section{Résumés}

Le présent article décrit le projet d'édition électronique d'un ensemble de gloses universitaires du XIII ${ }^{\mathrm{e}}$ siècle copiées autour des traités d'Aristote sur la nature dans leurs premières traductions arabo et gréco-latines. Aucune édition de référence n'a pu être réalisée sur ce corpus, bien que des éditions partielles aient été faites. D'un autre côté, les éditions de gloses en général, qu'elles soient sous format papier ou électronique, répondent de diverses manières aux attentes du lecteur : restitution de la complexité de la tradition manuscrite et du lien entre les gloses et le texte, facilité de lecture des gloses marginales et interlinéaires en séquence avec le texte. L'encodage en XML-TEI permet de rendre compte de ces aspects et de produire, via des feuilles de transformation XSLT, une sortie vers les formats papier et électronique, ce dernier autorisant de nouveaux modes de consultation des éditions de sources anciennes. À travers la création d'outils et de méthodologies d'encodage et de transformation, le projet vise la constitution d'un pôle de ressources pour l'édition scientifique.

The present paper describes a project aiming at editing University glosses of the thirteenth century copied around the Aristotelian treatises on nature. No reference edition of this corpus has been carried out. Furthermore, editions of glosses in paper or electronic form do not always meet the reader's needs, among which the rendition of complex manuscript traditions and of the link between the glosses and the text, or a simplified interface for reading the text and the marginal and interlinear glosses sequentially. XML-TEI encoding allows to render these features and to produce outputs to paper and electronic formats, thanks to XSLT transformation sheets. The electronic format enables the edi- 
tions of ancient sources to be read in new ways. Through the creation of encoding and transforming tools and methodologies, the project seeks the constitution of a resource center for scientific edition.

\section{Entrées d'index}

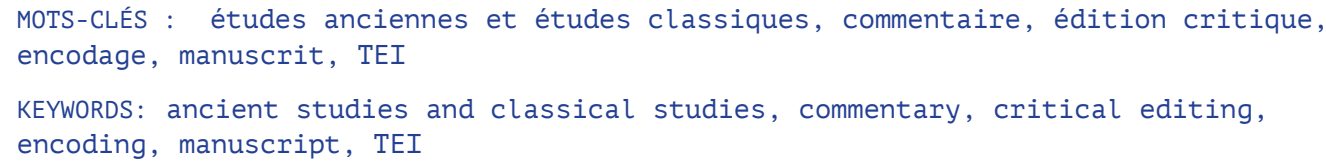

\section{Introduction et objectifs du projet}

Gloses et commentaires sont les formes par lesquelles l'Occident latin, au XIII ${ }^{\mathrm{e}}$ siècle, a reçu et assimilé la philosophie aristotélicienne, socle de la formation dispensée dans les universités européennes ${ }^{1}$.

La glose d'Oxford ou glossa Anglicana désigne un corpus de gloses présentes autour des textes des anciennes traductions des traités naturels aristotéliciens (ces traductions forment ce que l'on appelle le corpus vetustius $^{2}$ ) (Brams et Bossier 1990 ; Burnett 1996, 38-41 ; Burnett et Mendelsohn 1997) ${ }^{3}$. Ce corpus est attesté en particulier dans des manuscrits anglais, et semble avoir été constitué au sein de l'université d'Oxford au XIII $^{\mathrm{e}}$ siècle, dans le cadre des leçons sur les libri naturales - livres sur la nature - au programme de la faculté des arts, qui représentait le premier grade des études supérieures (Weijers et Holtz 1997 ; Lafleur et Carrier 1997).

L'objectif du présent projet, financé par l'Institut des sciences humaines et sociales du CNRS (2017-2020) et entrepris à l'Institut de recherche et d'histoire des textes (CNRS), est une étude pionnière, exhaustive et complète de la glossa Anglicana, à travers la recension de ses manuscrits et l'édition scientifique de la glose associée à deux des livres naturels aristotéliciens, le De plantis du pseudo-aristotélicien Nicolas de Damas ${ }^{4}$, et les Meteora ${ }^{5}$. L'un des résultats attendus est l'établissement de son édition et sa valorisation grâce à une mise en ligne permise par le balisage en XML-TEI ${ }^{6}$ de la structure de la glose. Le balisage en XML-TEI comprendra également les variantes des manuscrits retenus, et permettra une mise à disposition du texte établi, une consultation aisée de cette source, et des recherches croisées grâce à un moteur de recherche adapté.

Le traitement et la valorisation de cette source à la fois complexe, mal connue mais fondamentale pour la connaissance de l'histoire intellectuelle du XIII ${ }^{\mathrm{e}}$ siècle, permettront de créer, standardiser et diffuser un ensemble d'outils et de méthodologies pour l'édition scientifique en XML-TEI et son exploitation multimodale. 


\section{La glose d'Oxford}

\section{Matériau}

On trouve ces chaînes de gloses copiées dans les marges de ce que les Anglais appellent des "textbooks ", en quelque sorte des manuels comprenant l'ensemble des traités naturels d'Aristote dans les traductions anciennes qui composent le corpus vetustius, et qui sont en usage à partir de la première moitié du XIII ${ }^{\mathrm{e}}$ siècle : Physica (Physique), De celo et mundo (Du ciel), De generatione et corruptione (De la génération et de la corruption), Meteora (Météorologiques), De plantis (Des plantes), De anima (De l'âme), De memoria et reminiscentia (De la mémoire et de la réminiscence), De sensu et sensato (De la sensation et des sensibles), De somno et vigilia (Du sommeil et de la veille), De morte et vita (De la vie et de la mort, comprenant aussi les traités De la longévité et de la brièveté de la vie et De la jeunesse et de la vieillesse), De differentiis spiritus et anime (Sur la différence entre l'esprit et l'âme), De causis (Livre des causes), les deux derniers étant apocryphes, de même que le De plantis ${ }^{7}$. Ces manuscrits disposaient de larges marges, souvent divisées au moyen de la réglure, sur lesquelles les étudiants pouvaient copier cette glose sur plusieurs niveaux (voir figure 1).

FIGURE 1. DE PLANTIS GLOSÉ (MS. BRITISH LIBRARY, HARLEY 3487)

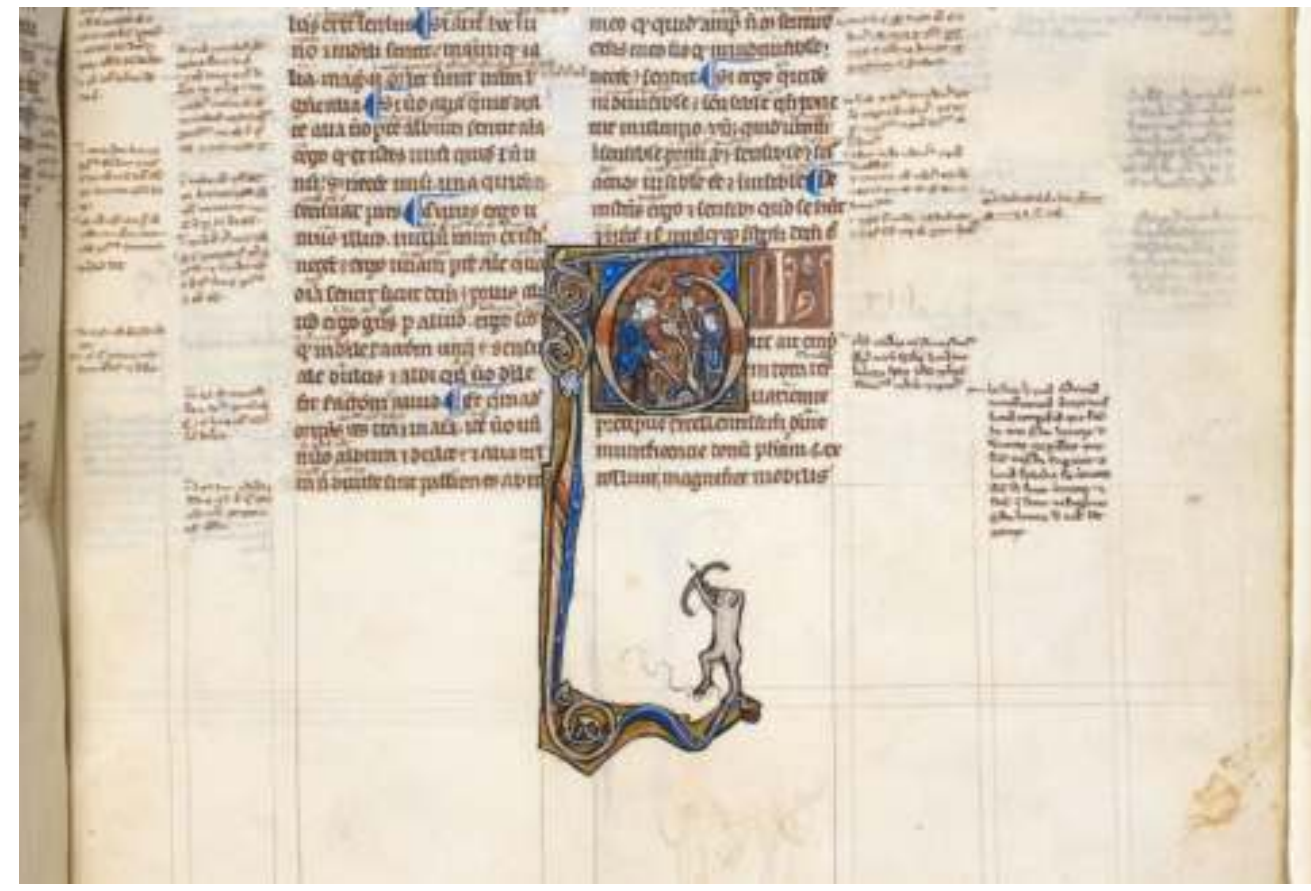

Début du De plantis glosé dans le ms. British Library, Harley 3487, $\mathrm{f}^{\mathrm{O}}$ 224r, vers 1275-1300. Source : http://www.bl.uk/manuscripts/FullDisplay.aspx?ref=Harley_MS_3487.

Les gloses pouvaient être liées au lemme, c'est-à-dire au terme glosé, par un système de signes de renvoi. Dans d'autres cas, le lemme était reproduit au début du texte de la glose et souligné. Le texte d'Aristote était 
copié dans un module plus gros, et avec un interligne suffisamment grand pour permettre l'insertion de gloses interlinéaires, de façon analogue à la structure de la glose ordinaire de la Bible.

\section{État des lieux}

\section{Corpus}

La question de la cohérence de cette chaîne de gloses a été soulevée par Fernand Bossier et Jozef Brams dans leur édition de la Physica. Ensuite, c'est l'article de Charles Burnett de 1996 qui a mis en lumière l'existence de ce corpus. En croisant les informations contenues dans cet article avec les différentes éditions de la glose réalisées depuis ${ }^{8}$, on obtient une liste de 26 manuscrits potentiels porteurs d'un état de la glose d'Oxford sur au moins un traité du corpus vetustius ${ }^{9}$. En effet, l'état des gloses, mais aussi leur présence sur tel ou tel traité, divergent d'un manuscrit à l'autre, d'où la nécessité de réaliser en premier lieu une typologie des corpus de gloses (corpus étendu, corpus abrégé, présence de commentaires complets en glose comme ceux d'Adam de Buckfield, maître ès arts d'Oxford actif vers le milieu du XIII ${ }^{\mathrm{e}}$ siècle et premier commentateur de l'intégralité du corpus aristotélicien sur la nature [Long 2013, 1-14 ; Edmund J. French 1998 ; Donati 1998, 111-114...]), ainsi qu'une identification systématique d'autres témoins, en partant du répertoire de manuscrits de l'Aristoteles Latinus (Lacombe et al. 1939, 1955 ; Minio Paluello 1961) et des catalogues de commentaires aristotéliciens comme ceux de Rodney Thomson (2011, 2013), qui décrivent les manuscrits conservés sur le sol anglais. D’autres manuscrits anciens sont conservés ailleurs en Europe. C. Burnett parlait de treize manuscrits du corpus vetustius datés du XIII ${ }^{\mathrm{e}}$ siècle et signalés par le catalogue de l'Aristoteles Latinus pour les bibliothèques anglaises, parmi lesquels neuf portent une version de la glossa Anglicana et quatre autres une glose non identifiée. Le lien entre corpus vetustius et corpus glosé devra être éclairci et daté.

En ce qui concerne le De plantis, neuf manuscrits glosés sont signalés dans l'édition de la glose d'Oxford sur ce traité par Roger Kenneth French (1997a), mais l'éditeur précise qu'il ne s'agit que des manuscrits anglais ${ }^{10}$. L'édition de French prend en compte les $n^{0} 1-2,6-7,15-18,23$ de la liste fournie en annexe. Il est à noter que dans le témoin de la glossa Anglicana le plus ancien connu, le manuscrit Vaticano, Urb. lat. 206 ( $\left.{ }^{\circ} 4\right)$, le texte du De plantis n'est pas glosé. Concernant les Meteora, il n'existe à notre connaissance aucune étude spécifique de la glossa sur ce traité.

Le De plantis, bien qu'apocryphe, est un maillon essentiel dans la classification aristotélicienne des sciences qui forme l'épine dorsale de l'enseignement universitaire en philosophie au XIII $^{\mathrm{e}}$ siècle, puisqu'il contient l'essentiel de ce qui est considéré à l'époque comme le savoir botanique aristotélicien. Dans cette classification, la science théorique ou spéculative recouvre les domaines suivants : métaphysique, science naturelle ou physique, et sciences mathématiques. La science naturelle d'Aristote s'occupe des corps mobiles. Parmi eux, au sein des corps "générables et corruptibles ", après les corps inanimés traités dans les $M e$ teora, l'âme végétative, qui est celle des plantes, est traitée en premier parmi les corps animés. Elle est suivie de l'âme sensible, celle des ani- 
maux, qui fait l'objet du De animalibus, et de l'âme rationnelle, abordée dans le De anima et les petits traités ou opuscules constituant les Parva naturalia (De sensu et sensato, De memoria et reminiscentia, De somno et vigilia, De morte et vita $\left.{ }^{11}\right)$.

Comparé aux grands traités qui circulent avec lui dans les "textbooks" comme la Physica ou le De celo et mundo, le De plantis paraît cependant de taille relativement modeste. L'édition du De plantis dans la collection de l'Aristoteles Semitico-Latinus totalise 46 pages (Drossaart Lulofs et Poortman 1989, 515-561). Dans les témoins glosés, le De plantis représente une douzaine (ms. Harley $3487, \mathrm{n}^{0} 18$ ) à une vingtaine de feuillets (ms. Royal 12.G.2, $\mathrm{n}^{\mathrm{O}}$ 15). Le De plantis compte deux livres. Pour le premier livre, je partirai de l'édition des gloses marginales par R. K. French (35 p.), tout en complétant son travail par la transcription des gloses interlinéaires et l'inclusion des variantes des manuscrits continentaux qui auront été retenus. En ce qui concerne le deuxième livre, plus court, tout reste à faire.

Les Meteora représentent un texte environ deux fois plus long que le De plantis : 33 feuillets dans le ms. $\mathrm{n}^{\mathrm{O}} 18$, une cinquantaine dans le ms. $\mathrm{n}^{\mathrm{O}} 15$.

R. K. French, dans son édition des gloses sur le livre I du De plantis, indique avoir repéré plusieurs états de la glose sur le De plantis :

- un état qu'il considère comme complet, représenté par 5 manuscrits $\left(\mathrm{n}^{0} 6,16,18,2,23\right.$ de la liste)

- un état légèrement abrégé, et comptant des gloses supplémentaires, représenté par un seul manuscrit $\left(\mathrm{n}^{0} 15\right)$

Comme mentionné plus haut, l'édition de R. K. French ne prenait en compte que des manuscrits anglais. D’autre part, deux des témoins considérés par R. K. French comme portant un état complet de la glose sur le De plantis sont décrits par C. Burnett comme contenant un état abrégé de la glose. Mais C. Burnett s'appuyait sur une analyse globale des exemplaires du corpus vetustius qu'il avait intégrés dans son étude. Il est donc primordial de raisonner traité par traité pour créer une typologie des gloses, c'est-à-dire en deçà du niveau du manuscrit.

\section{Datation}

Il est difficile de dater précisément la genèse du corpus des gloses. Nous disposons cependant d'un certain nombre de termini. Iolanda Ventura a montré que Barthélemy l'Anglais employait la glossa Anglicana au De plantis dès les années 1240 dans le livre XVII de son De proprietatibus rerum (Ventura 2007, XV). J'ai pu démontrer dans ma thèse que le texte de l'anonyme Compilatio de libris naturalibus Aristotelis ou Compendium philosophie, du milieu du XIII $^{\mathrm{e}}$ siècle, employait des passages de cette glose $^{12}$.

Selon C. Burnett, le manuscrit Vaticano, Urb. lat. $206\left(\mathrm{n}^{0} 4\right.$ de la liste), dont le texte central a été copié avant 1253, pourrait représenter un état précoce de la glossa Anglicana (Burnett 1996, 40) mais, d'après les termini donnés ci-dessus, il faudrait encore remonter la date présumée de constitution du corpus, peut-être dans un état primitif, aux années 1240 voire 1230. Il est évident que de nouvelles enquêtes dans ce domaine sont nécessaires et que l'identification d'autres utilisateurs de la glose pourra permettre de préciser les choses et sera facilitée par la future édition électronique des gloses. 


\section{Sources}

Grâce aux études de C. Burnett et R. K. French notamment, on sait que la glose reprend les commentaires d'Averroès, d'Alfred de Shareshill, mais aussi plus ponctuellement des définitions empruntées à Isidore de Séville. En ce qui concerne le commentaire sur le De plantis d'Alfred de Shareshill, l'étude de R. K. French a montré que la glose en transmettait un contenu parfois plus riche que les témoins livrant le texte sous la forme d'un commentaire indépendant tel qu'il a été édité par R. J. Long (Burnett 1996, 21-50 ; R. K. French 1997, 223-251 ; Long 1985, 125-167).

Certaines gloses livrant un contenu médical ou pharmaceutique font directement référence à Avicenne ${ }^{13}$. D'autre part, il est intéressant de trouver des ajouts ponctuels, comme des mises à jour du savoir, dans certains témoins. J'ai retrouvé une citation attribuable à Albert le Grand, dans la glose présente dans le ms. Harley $\left(n^{0} 18\right)$, du troisième quart du XIII $^{\mathrm{e}}$ siècle et peut-être copié à Oxford ${ }^{14}$. Cette glose ne fait pas partie du corpus de la glossa Anglicana tel qu'on peut le voir dans les autres manuscrits. Il pourrait donc s'agir là d'un ajout au corpus standard à partir de la lecture d'un auteur récent, voire contemporain, qui écrit dans les années 1254-1257.

Plusieurs chercheurs ont mis en évidence une influence d'Adam de Buckfield sur la glose, mais il est possible qu'Adam lui aussi emploie un corpus glosé, hypothèse qui pourra être confirmée grâce à la mise en ligne de l'édition de la glose ${ }^{15}$. On a donc affaire à un corpus ouvert et en constante mutation. Dans certains manuscrits, le commentaire d'Adam figure en glose continue, que ce soit en marge inférieure ou en glose encadrante. Il faudra prendre en compte cette donnée dans l'établissement du texte de la glose et veiller à fournir des critères permettant une distinction entre glose et commentaire continu ${ }^{16}$.

\section{Production et réception}

L'un de ces annotateurs, Henry de Rainham, indique dans une note de sa main du dernier quart du XIII ${ }^{\mathrm{e}}$ siècle, qu'il a glosé et amendé le texte tout en écoutant les leçons :

London, British Library Royal 12. G. II, f. $2 \mathrm{r}$ : Quem librum scripsit Henricus de Renham et audivit in scolis Oxonie et emendavit et glosavit audiendo.

Il semble plus probable, d'après l'aspect généralement soigné des gloses, que celles-ci aient été mises au propre après coup à partir de notes prises au cours des leçons. La comparaison entre les différents témoins de la tradition manuscrite montre un texte vivant, qui peut dans certains cas donner des signes d'erreurs d'interprétation peut-être dues à des reports trop hâtifs ${ }^{17}$. Le caractère vivant et renouvelé du texte est aussi attesté par l'identification des sources des gloses comme le montre l'exemple de la citation tirée d'Albert le Grand dans le manuscrit Harley.

Cette glose est donc une source fondamentale pour notre connaissance de la vie intellectuelle et de l'enseignement universitaire au XIII $^{\mathrm{e}}$ siècle $^{18}$. L'étude comparative des divers états de la glose donnera le moyen de connaître son évolution et d'observer d'éventuelles couches d'inclusion de citations d'auteurs précis ou d'identifier des utilisateurs de la glose, ce qui pourra permettre d'établir une chronologie relative des différents états du texte. 


\section{État des éditions}

\section{Liste des éditions}

La liste des gloses éditées est la suivante :

- 1997 : C. Burnett, gloses sur la Physica I, 4 manuscrits anglais (Burnett et Mendelsohn 1997)

- 1997 : R. K. French, gloses marginales (donc sans les interlinéaires) sur le De plantis I, 9 manuscrits anglais (R. K. French 1997a)

- 1998 : Edmund John French, gloses sur le De differentia spiritus et anime, 1 manuscrit (E.J. French 1998, 202-276)

- 2008 : Griet Galle, gloses sur le De sensu et sensato I, 10 manuscrits (Galle 2008)

- Voici la liste des traités composant le corpus vetustius telle que donnée par C. Burnett dans son article de 1996 :

- Physica

- De celo et mundo

- De generatione et corruptione

- Meteora ${ }^{19}$

- De plantis

- De anima

- De memoria et reminiscentia

- De sensu et sensato

- De somno et vigilia

- De morte et vita

- De differentiis spiritus et anime

- De causis

\section{Problèmes posés par les éditions de la glose d'Oxford}

D’après les éléments donnés ci-dessus, il est évident que les éditions soit ne concernent qu'un livre, soit ne prennent en compte qu'une partie des manuscrits, soit les deux. De plus, la plupart des traités n'ont reçu aucune édition de leurs gloses.

Dans un autre registre, on rencontre dans ces éditions un problème de lecture et d'accès aux gloses. La reproduction de la mise en page originale étant chose à peu près impossible à réaliser dans une édition moderne, il faut, en tant qu'éditeur, faire des choix, ce qui suppose de renoncer à certains aspects, qu'ils soient éditoriaux ou scientifiques. Passons en revue les choix qui ont été faits par les éditeurs mentionnés ${ }^{20}$.

Dans l'édition de C. Burnett des gloses sur la Physica, le texte aristotélicien dans les traductions gréco-latine et arabo-latine est présenté en début de section selon la segmentation d'Averroès, car l'éditeur n'a repris que les gloses se référant à ce commentateur, pour mettre en valeur la compilation du matériel averroïste par le glossateur. À la fin du paragraphe, les gloses sont éditées dans la colonne de droite, tandis que le texte d'Averroès est donné pour comparaison dans la colonne de gauche. Le texte aristotélicien (translatio vetus) est donné d'après l'édition de l'Aristoteles Latinus avec les variantes principales, avec en complément le texte de la traduction de Michel Scot qui accompagne l'édition du com- 
mentaire d'Averroès. On voit qu'un effort a été fait pour mettre en regard les lieux parallèles, mais cet effort se heurte aux limites du papier, dans une édition qui reste partielle puisque circonscrite aux gloses émanant d'une source en particulier.

Dans l'édition de R. K. French des gloses sur le De plantis, tout le texte central aristotélicien est reproduit en une première section à partir d'un seul manuscrit sans apparat, puis les gloses sont éditées à la suite du texte avec un apparat. Dans les deux sections, les lemmes sont soulignés. La lecture du texte glosé est rendue plus difficile puisque, pour lire le texte en séquence, il faut a minima avoir une page de la première section ouverte à côté de la page correspondante dans la section des gloses.

Dans l'édition d'E. J. French des gloses sur le De differentia spiritus et anime, le texte central n'a pas été reproduit, et on dispose uniquement de la transcription d'un manuscrit. L'éditeur fait ponctuellement une référence au fait que d'autres témoins ont des gloses plus étendues, sans précision supplémentaire. Il s'agit là davantage d'un outil de travail que d'une édition, ce qui est d'ailleurs clairement notifié par l'éditeur dans le préambule.

Dans l'édition de G. Galle des gloses sur le De sensu et sensato I, on a fait en sorte de restituer avec précision la place de chaque élément et d'indiquer les différences de localisation des gloses (migrations de la marge à l'interligne ou inversement ; changements de lemme) d'un manuscrit à un autre. L'ensemble peut se lire en séquence grâce à la division du texte d'Aristote présente dans certains manuscrits et héritée du commentaire d'Adam de Buckfield. Le texte aristotélicien est transmis d'après la translatio vetus, dont G. Galle indique être en train de réaliser l'édition, et qu'elle accompagne d'un apparat critique qui comprend les variantes du texte central et des gloses. La lecture du texte et des gloses nécessite en revanche une familiarisation avec un certain nombre de signes et d'abréviations.

En conclusion, il ne semble pas y avoir de manière idéale d'éditer un texte glosé sur papier. G. Galle produit l'édition la plus précise et la plus scientifique, mais cette précision nuit quelque peu à la facilité de lecture.

\section{Le projet d'édition}

\section{Problématiques posées par l'édition de la glose}

\section{États de la glose et méthodologie critique}

Contrairement à la situation de la glose ordinaire dont on a pu dire qu'elle avait été " canonisée " assez tôt dans l'histoire de sa transmission, il est certain que la glose d'Oxford n'offrira pas un haut niveau de standardisation. Au sujet de l'apparat critique, Alexander Andrée indique que les éditeurs de la glose ordinaire de la Bible ont jusqu'ici trouvé plus pertinent d'indiquer la présence ou non des gloses plutôt que de collationner les variantes de leur texte (Andrée 2016, 6-8). Pour ce faire, il propose une approche "structurelle ", qui suppose en premier lieu d'établir un canon des gloses marginales et interlinéaires pour chaque livre, selon une méthode suivant plusieurs étapes : 
- Le manuscrit conservé le plus ancien doit être identifié et son corpus de gloses enregistré

- L'ensemble des gloses est collationné sur les autres manuscrits retenus, dans l'ordre d'ancienneté jusqu'à la première édition

- De ce fait, on peut suivre le développement du corpus de gloses à travers le temps et repérer les manuscrits qui forment des maillons intermédiaires du processus de fixation de la glose

On peut donc éditer le corpus de gloses sur la base de ce travail en fournissant les notes critiques en apparat, ce qui implique d'éditer un manuscrit de base avec les variantes en apparat plutôt que de reconstituer un hypothétique archétype.

Si le corpus de la glose d'Oxford n'offre pas ce niveau de standardisation, les remarques d'A. Andrée sur la non-pertinence de la reconstitution " lachmanienne " d'un texte archétypal sont d'autant plus vraies dans notre cas. Nous connaissons mal aujourd'hui les divers états existants de la glose, même si les travaux déjà menés laissent supposer un état abouti ou tout au moins développé de la glose dans la seconde moitié du XIII ${ }^{\mathrm{e}}$ siècle. Dans tous les cas, la qualification d'état "développé " ou " étendu » ne recouvre absolument pas une canonisation et une diffusion contrôlée comme c'est le cas pour la glose biblique. Il est donc vain, dans notre cas, d'espérer identifier le manuscrit le plus ancien pour en faire le texte de base, d'autant plus que la plupart des manuscrits ne peuvent être datés précisément, et, lorsqu'ils le sont, la date de copie du texte central ne peut fournir qu'un terminus post quem pour la copie des gloses.

D'autre part, les copies de la glose d'Oxford proviennent de sources multiples (les notes prises par les étudiants lors de lectures universitaires) et non d'un original unique. Partant, l'idée d'archétype est inopérante. Pour accomplir la tâche d'édition, comme il a été mentionné, il convient d'établir en premier lieu une typologie formelle du corpus de gloses par manuscrit et spécifique à chaque traité, à partir de l'Aristoteles Latinus et des catalogues, et des reproductions disponibles. Cette typologie permettra de se repérer dans le corpus des manuscrits témoins.

Une méthodologie critique claire, rigoureuse et précise est indispensable pour mener à bien ce projet sans se perdre dans les méandres de la transmission mouvante des gloses. Une fois la typologie du corpus de gloses établie pour chaque manuscrit, on pourra, pour chaque traité, décrire des états principaux de la glose, et choisir un manuscrit de base pour chaque état principal recensé. Il paraît ensuite intéressant de reprendre une partie de la méthodologie proposée par A. Andrée et d'enregistrer la structure de la glose pour le manuscrit de base de l'état le plus étendu, ce qui suppose d'identifier et de caractériser précisément chaque glose et de lui attribuer un identifiant unique qui permettra d'indiquer si elle apparaît ou non dans les autres états manuscrits recensés.

\section{Statut du texte central}

Bien évidemment, le statut du texte central représente une question cruciale, notamment en regard des droits de reproduction des œuvres éditées.

Dans l'édition des gloses sur les livres I-II du De nuptiis de Martianus Capella, l'éditrice a transcrit les gloses avec le lemme, mais sans le texte principal, dans la mesure où celui-ci a reçu une édition critique récente, 
à laquelle elle fait référence (O'Sullivan 2010). Elle fournit donc un apparat critique pour les lemmes du texte de base, et un autre pour les gloses. Elle procède de la même façon dans son édition des gloses sur la Psychomachia de Prudence (O'Sullivan 2004). On fait référence à l'édition du texte central également dans l'édition des gloses sur le De institutione musica de Boèce (Bernhard et Bower 1993). Dans l'édition de l'Historia apostolica d'Arator, l'éditeur a rejeté dans un second volume l'édition des gloses latines et germaniques sur le texte (Orbán 2006). Le texte central est encore absent de l'édition des Scholia recentiora sur Juvénal, et de l'édition du Commentum Monacense sur Térence (Grazzini 2011 ; San Juan Manso 2015).

Il semble donc que le texte central pose un problème récurrent aux éditeurs, que ce soit dans le domaine du droit d'auteur ou de la mise en page, problème qu'il est plus commode d'éluder en ne reproduisant pas le texte ou en le reproduisant de manière séparée. Au regard des réserves que posent les éditions précédentes de la glossa Anglicana, qui ont été évoquées plus haut, et de manière générale les éditions de gloses ne reproduisant pas le texte central, il serait intéressant d'y donner accès avec les gloses qui lui sont attachées, d'autant plus si on veut proposer au lecteur, non uniquement un ensemble de données scientifiques (l'édition des gloses), mais en réalité un texte à lire. Dans ce cas et compte tenu des problématiques juridiques évoquées, il faut donc choisir entre :

- Trouver un accord avec les éditeurs pour employer le texte édité sans l'apparat; dans le cas du De plantis et des Meteora, il s'agit des éditeurs de l'Aristoteles Semitico-Latinus chez Brill

- Proposer la transcription du texte central de notre manuscrit de base ; dans ce cas, il faudra probablement collationner le texte avec celui de l'édition dans l'Aristoteles Semitico-Latinus

La seconde solution présente certains avantages, en offrant la possibilité d'être rapidement réalisée en récupérant ou en transcrivant le texte de l'édition et en encodant les variantes en XML-TEI de sorte à reconstituer automatiquement le texte du manuscrit de base, au moyen d'un outil comme la Versioning Machine ou de l'outil dédié à l'affichage de versions parallèles dans la Critical Apparatus Toolbox réalisée par Marjorie Burghart ${ }^{21}$. Dans tous les cas, ce choix devra être expliqué dans l'avertissement au lecteur.

\section{Segmentation du texte et mise en page}

Le problème de la segmentation du texte central a suscité beaucoup de réflexion, principalement chez les éditeurs qui ont choisi de mettre en page texte central et gloses côte à côte. Comme on l'a vu, des solutions divergentes ont donc été mises en place pour le papier par C. Burnett et G. Galle. D'une manière plus générale, la question de l'étude et de l'édition des gloses connaît un regain d'intérêt récent ${ }^{22}$ après des décennies d'omission des questions autres que lexicographiques dans ce domaine. La difficulté d'accès au matériau (difficulté de lecture, corpus fragile) et la transmission mouvante des gloses, empêchant leur édition, y sont sans doute pour quelque chose (Teeuwen 2011, 19-20).

Cependant il ne semble pas exister de méthodologie idéale pour éditer un texte glosé. Les limites de l'édition d'un unique manuscrit sont décrites par Mariken Teeuwen, bien qu'elle ait choisi cette solution pour 
son édition électronique des gloses sur le De nuptiis de Martianus Capella (voir plus loin). Elle met aussi justement en exergue les inconvénients d'une édition critique des gloses qui reproduirait un texte central reposant sur une édition et présentant donc un caractère " lissé " non attesté dans les manuscrits, qui aboutirait par conséquent à une édition " éclectique " (Teeuwen 2007, 203). Cependant, cette situation existe déjà dans les manuscrits, puisque, dans certains témoins du corpus de la glossa Anglicana, on trouve des gloses portant sur des lemmes qui sont absents du témoin en question, ce qui induit une dissociation entre le texte central et les gloses ${ }^{23}$.

\section{Les textes glosés : les éditions papier}

Dans le domaine de l'édition papier, les possibilités offertes par le langage de composition LaTeX $^{24}$ en matière de mise en page d'éditions dotées d'apparats multiples, y compris marginaux ${ }^{25}$, devront être explorées. LaTeX permet en outre d'obtenir des apparats critiques incluant la numérotation automatique des lignes auxquelles font référence les notes de bas de page, de multiplier les apparats de notes de façon harmonieuse sur la page et de réaliser des éditions synoptiques.

On peut se pencher sur le cas de la glose ordinaire de la Bible, dont la reproduction du texte central pose bien évidemment moins de problèmes juridiques que dans les éditions que nous venons d'évoquer. Les incunables, comme la célèbre édition de Strasbourg par Adolf Rusch de 1480-1481 ${ }^{26}$, reproduisent la mise en page des manuscrits avec gloses interlinéaires entre les lignes du texte biblique, donné en gros module, et gloses marginales encadrantes. Au XIX ${ }^{\mathrm{e}}$ siècle, l'abbé Migne fournit une édition interpolée et incomplète puisque les gloses interlinéaires ne sont pas éditées (Migne 1852). De plus, le texte biblique n'est pas reproduit en dehors du lemme et on ne dispose d'aucun apparat critique. Dans les dernières décennies, différentes méthodologies ont été explorées pour éditer la glose biblique sur papier :

- Mark Allen Zier a réalisé une édition du début du livre de Daniel (Zier 1993). Le texte biblique est placé sur un côté de la page, dans un grand module et en gras. Les gloses sont éditées sur le côté du texte, de façon parallèle. Il n'y a aucune distinction entre glose marginale et glose interlinéaire.

- Mary Dove est la seule à avoir produit l'édition critique d'un livre complet de la Bible : le Cantique des cantiques (Dove 1997). Le texte biblique est imprimé en gras dans un encadré et segmenté en passages de quelques mots. Les gloses sont éditées à la suite, en distinguant les gloses interlinéaires des gloses marginales, qui voient leur lemme mis en gras. On trouve en bas de page un apparat des sources et un apparat critique. L'ensemble est édité de manière synoptique, en regard de la traduction.

- A. Andrée a édité le texte du premier livre des Lamentations, en plaçant le texte biblique en tête de page, en capitales (Andrée 2005). Les gloses interlinéaires sont données immédiatement à la suite, et sont liées à leur lemme par un appel de note. Les gloses marginales sont disposées en dessous des gloses interlinéaires, le lemme étant placé en capitales. Apparat des sources et apparat critique sont disposés en bas de page. Là encore la double page permet d'accéder à la traduction en regard. 
Dans ces derniers cas, l'apparat critique concerne aussi bien le texte central que les gloses.

Toutes ces mises en page sont fonctionnelles et remplissent leur office. Si on voulait améliorer les choses, on pourrait dire qu'à la lecture, bien plus que les gloses marginales qui reproduisent le lemme et donnent à lire un énoncé complet en soi, ce sont les gloses interlinéaires et leur mise en page qui peuvent poser problème ou tout au moins éloigner le lecteur de l'immédiateté de la lecture permise par la mise en page médiévale. Étroitement liées au terme dans son contexte, leur lecture compréhensive est plus difficile lorsqu'elles sont séparées du texte central.

\section{Les textes glosés : les éditions électroniques}

En ce qui concerne la publication électronique, plusieurs méthodologies ont été explorées, plus particulièrement dans le domaine des gloses du haut Moyen Âge.

- Dans l'édition des gloses sur Orose du manuscrit de Saint-Gall par Heidi Eisenhut (Universität Zürich ${ }^{27}$ ), les éléments suivants sont mis à disposition :

- en première colonne, une typologie des gloses (correction, glose lexicale, glose grammaticale, glose syntaxique, commentaire) - en seconde colonne, une localisation de la glose se référant à la page et à la ligne

- on a choisi de ne pas reproduire le texte central (hormis le lemme) et de renvoyer à l'édition moderne du texte d'Orose via un lien hypertexte

- en dernière colonne, on signale les témoins du texte d'Orose dans lesquels chaque glose est attestée

- l'apparat critique des gloses, qui signale aussi des variantes du lemme dans le texte de base et donc dans des manuscrits non glosés, apparaît au survol dans une infobulle et peut être fixé dans l'espace supérieur de l'interface

- les différentes mains à l'œuvre sont signalées par des couleurs différentes dans la transcription des gloses

- un moteur de recherche donne la concordance du terme recherché dans les gloses ou dans le corpus des sources interrogées sur le Corpus corporum ${ }^{28}$, qui a par ailleurs été développé par les mêmes informaticiens

- l'apparat des sources est donné dans des notes de bas de page qui apparaissent, dans l'espace supérieur de l'interface de consultation, au clic sur l'appel de note dans le texte

- l'ensemble est proposé en lecture synoptique avec le fac-similé du manuscrit disponible sur $e$ - codices $^{29}$

- Dans l'édition des gloses sur Martianus Capella du manuscrit de Leyde par M. Teeuwen (Huygens Instituut, La Haye ${ }^{30}$ ), l'interface de consultation est divisée en quatre colonnes ("panels») interchangeables et supprimables, qui proposent de consulter :

- la structure de l'édition sous forme d'arborescence

- le fac-similé du manuscrit via la reproduction d'un microfilm en noir et blanc

- l'édition «semi-diplomatique » du texte central, dotée de notes qui donnent le texte du lemme et le contenu de la glose au survol dans 
une infobulle

- la liste des gloses avec la restitution du lemme

- un moteur de recherche simple

L'idée des panels est intéressante mais pas particulièrement facile à utiliser, surtout lorsque l'on souhaite agrandir l'image pour lire les gloses. De plus, le lien est à sens unique entre le panel de la transcription et celui des gloses, de sorte que si l'on suit la colonne des gloses, bien plus longue, on se trouve vite déconnecté du panel du texte central. Il n'y a pas ici d'apparat critique ni d'apparat des sources, mais des remarques concernant des variantes d'autres manuscrits ou l'identification de sources peuvent occasionnellement être ajoutées à la transcription des gloses. Il n'y a pas non plus d'identification de changements de main dans la transcription des gloses.

- Dans l'édition des gloses sur Priscien du manuscrit de Saint-Gall par Pádraic Moran (National University of Ireland, Galway ${ }^{31}$ ), le lecteur accède, via des colonnes successives, à :

- la page et la ligne de lédition moderne du texte de base de Priscien, avec un lien hypertexte pour en ouvrir la version numérisée - la localisation précise de la glose dans le manuscrit (page et ligne), avec un lien vers le fac-similé

- une typologie très détaillée des gloses, avec un lien permettant d'afficher toutes les gloses de ce type

- la transcription des gloses comprenant la restitution du lemme et avec, le cas échéant, un lien hypertexte vers l'analyse du terme vieil irlandais. La présence des gloses dans d'autres manuscrits est signalée dans le texte avec une référence au feuillet et à la ligne - un moteur de recherche permettant d'effectuer une recherche textuelle dans les gloses ou le texte central tout en filtrant par référence de la glose, par type ou par livre

- Dans le projet Gloss-e d'édition électronique de la glose ordinaire de la Bible par Martin Morard (IRHT, Paris ${ }^{32}$ ), pour pouvoir donner à lire le texte dans sa continuité, on a choisi la solution d'un niveau important de segmentation du texte. Ainsi, on a pu placer les gloses interlinéaires sur le bon terme sans répéter le terme glosé ni placer de note, et offrir une organisation continue du texte central et des gloses. L'usage de la couleur et d'une taille de police importante joue un grand rôle dans la lisibilité de l'ensemble, ce qui peut éventuellement poser des problèmes dans une édition papier. L'interface de consultation donne à voir :

- le texte biblique fortement segmenté, entrecoupé par les gloses qui le concernent - des notes indiquant des variantes dans l'édition Weber du texte biblique et ponctuellement des variantes manuscrites - des notes d'identification des sources

Il est à noter que le format d'encodage des données est le XML-TEI, obtenu grâce à une transformation du format Word stylé.

- Dans l'édition des gloses sur Martianus Capella du manuscrit de Cologne par Monika Isépy et Bernd Posselt (LMU, Munich ${ }^{33}$ ), l'outil de consultation fondé sur Java permet d'accéder à :

- l'arborescence de l'édition dans la fenêtre de gauche

- dans l'espace restant, deux fenêtres pour afficher au choix, via des 
onglets :

* le texte de Martianus

* la transcription des gloses avec le lemme correspondant, qui est lié au texte central par un lien hypertexte

* les lieux correspondants dans les éditions des commentaires de Rémi d'Auxerre et de Jean Scot Érigène

* les passages marqués en vert dans les gloses permettent d'indiquer des corrections éditoriales, qui apparaissent au survol dans une infobulle

- en partie inférieure de l'interface de consultation, le fac-similé du manuscrit doté de zones cliquables correspondant à chaque glose

Il est intéressant de noter qu'ici textes et gloses sont liés à des zones de l'image et inversement, ce qui permet leur consultation interactive. En revanche, on ne dispose pas non plus d'un apparat critique ni d'un apparat des sources.

- Dans l'édition, par Martin Hellmann, Stefan Ullrich et Claudia Wiener, au sein de la même équipe (Munich), des gloses sur Perse ${ }^{34}$, l'interface de consultation est divisée en deux colonnes, qui permettent de consulter au choix :

- le texte de base de Perse

- le texte des différents manuscrits, livrant le texte des gloses au sein (ou non) du texte de base du manuscrit choisi ; les gloses sont affichées en caractère normal, tandis que le texte de base s'affiche en gras ; un jeu de couleurs permet de distinguer les lemmes, les corrections éditoriales, les abréviations développées, etc.

Tous les textes sont liés par la référence du vers, qui permet d'afficher le lieu correspondant dans l'autre colonne. Chaque texte peut être affiché avec ou sans les corrections éditoriales et les identifications de sources, qui s'affichent dans le texte indifféremment. On note que l'on ne donne pas accès à un apparat critique unifié, mais, au contraire, on permet d'afficher les textes des différents manuscrits séparément.

Ces deux réalisations ne semblent pas mettre à disposition un moteur de recherche.

En revanche, les deux projets, menés au sein de la même unité, sont les seuls à avoir publié une méthodologie d'encodage des textes glosés fondée sur un schéma conforme au langage XML-TEI ${ }^{35}$. Cependant, cette méthodologie n'inclut pas l'encodage de l'apparat critique.

Dans le registre des glossaires, proches par le contenu mais éloignés par la forme, on peut citer la réalisation récente de l'édition du Liber glossarum par Anne Grondeux et Franck Cinato (HTL, université Paris-Diderot) ${ }^{36}$. La méthodologie détaillée sur le site ${ }^{37}$ indique que le format XML-TEI des fichiers actuels a été obtenu à partir d'une transformation de fichiers tableurs, ce qui est permis par la structure du texte plus ou moins stéréotypée. Les notes d'apparat critique sont liées au texte en suivant la «location-referenced method» détaillée dans les Guidelines de la $\mathrm{TEI}^{38}$, c'est-à-dire après le contenu de la glose, avec un lien au texte réalisé grâce à des attributs pointeurs, plutôt que dans le texte. La pérennité de l'édition est garantie par l'archivage sur la plateforme Huma-Num ${ }^{39}$.

Le choix de la TEI pour l'encodage du texte paraît le seul à même de permettre la réalisation d'une édition de texte glosé alliant la description de la structure du corpus glosé, le lien vers d'autres textes et l'encodage 
des variantes de sorte à pouvoir afficher le texte de l'un ou l'autre manuscrit. De même, l'encodage des mains et des formes normalisées permet de consulter le texte dans sa forme originale ou régularisée/corrigée, tout en autorisant des éditions synoptiques et une transformation vers un autre format adapté au papier ${ }^{40}$. De plus, le format ouvert et standardisé XML-TEI laisse espérer une plus grande pérennité des données dans le temps. Sur le site de e-laborate, l'outil d'édition collaborative déjà évoqué développé au Huygens Instituut, c'est le choix qui est recommandé lorsque l'éditeur souhaite réaliser une édition synoptique ou pourvue d'annotations critiques importantes ${ }^{41}$.

Enfin, il paraît indispensable, de nos jours, de mettre à disposition les fichiers sources XML ${ }^{42}$ afin qu'ils puissent être réutilisés dans d'autres perspectives, par exemple pour l'analyse textométrique réalisée avec des outils comme la plateforme $\mathrm{TXM}^{43}$.

Rendre leur place aux gloses marginales et interlinéaires dans l'organisation de la page représente un véritable défi technique et éditorial. La méthodologie d'encodage retenue devra prendre en compte de façon réaliste les possibilités de mise en page du papier et d'affichage électronique. En d'autres termes, l'ensemble du processus et des solutions techniques adoptées doit être conceptualisé avant de commencer l'encodage.

\section{Méthodologie technique}

\section{Modalités d'encodage du texte}

\section{Réalisation d'un schéma XML-TEI et intermédiaire de LaTeX}

La première tâche technique a été la définition d'un schéma XML-TEI permettant de représenter la complexité de la structure de la glose tout en identifiant les gloses équivalentes d'un manuscrit à l'autre, mais aussi de restituer les variantes de la tradition manuscrite des gloses et du texte central, y compris dans le lien de chaque glose au lemme, lien qui varie selon le manuscrit. La seule solution qui remplissait tous ces critères, et notamment la restitution du caractère mobile des gloses, était celle d'un encodage en stand-off aboutissant à un fichier contenant le texte central et un autre fichier constituant la bibliothèque des gloses. Les deux fichiers sont liés au moyen d'attributs pointeurs portés par le lemme dans le fichier du texte central, qui renvoient vers l'identifiant unique de la glose dans le fichier des gloses.

Ce schéma doit permettre la transformation ultérieure vers les formats Web et papier. Dans ce dernier domaine, l'accent doit être mis sur une réflexion sur la transformation vers le langage de composition de documents LaTeX.

Pour ce faire, une solution envisageable est l'encodage double des variantes en XML-TEI sur le même fichier source :

- une fois en notes pour permettre la création d'un apparat critique traditionnel destiné à l'impression via la transformation vers LaTeX

- une fois en suivant la méthode "parallel segmentation» décrite dans les Guidelines de la TEI ${ }^{44}$, qui permet de centraliser l'encodage de tous les lieux variants, et ensuite de reconstituer le texte de chaque 
manuscrit en utilisant un outil comme la Versioning Machine ${ }^{45}$ pour transformer le fichier.

Ce double encodage permet de contourner les problèmes liés à la transformation ultérieure du XML et d'atteindre l'objectif de l'affichage multimodal : c'est ce qui a été fait par le pôle Document numérique de la MRSH de Caen sur le texte de la Chronique de Malaterra édité par MarieAgnès Avenel ${ }^{46}$ : il est possible d'afficher le texte latin de base et son apparat critique traditionnel en regard de la traduction française, ou le texte de chaque manuscrit séparément, avec des options d'affichage ou non de l'encodage TEI que l'on peut expérimenter aussi par ailleurs dans les $B V H^{47}$ ou dans Gloss-e. D'autre part, le même fichier source a été utilisé pour la production de l'édition papier.

Une autre voie est offerte par les outils de conversion de XML-TEI à LaTeX prenant en charge l'encodage selon la méthode "parallel segmentation " des Guidelines de la TEI. Citons dans ce registre l'outil "Print an edition " de la TEI Critical Apparatus Toolbox déjà évoquée, dont la feuille de transformation XSLT est éditable, ce qui permet d'adapter la transformation à la structure du texte d'entrée ${ }^{48}$. Une réflexion reste à mener sur l'adaptation de cette feuille de transformation à la structure particulière de l'encodage mis en œuvre sur le texte de la glose d'Oxford, et notamment de l'encodage en stand-off.

Envisager cette seconde solution présente de multiples avantages : économie de l'encodage mais aussi, pour rendre cette transformation possible, une contrainte plus forte sur cet encodage, ce qui garantit son homogénéité dans le temps et laisse espérer une plus grande interopérabilité.

Dans le domaine de la publication électronique, plusieurs solutions ont été envisagées. Premièrement, il était possible de réutiliser le moteur d'affichage générique MaX développé par le pôle Document numérique de la $\mathrm{MRSH}$ de Caen ${ }^{49}$ et mis à disposition, avec d'autres ressources, sur le portail de l'Equipex Biblissima ${ }^{50}$. Une autre solution était de laisser à un développeur le choix de l'approche à adopter en fournissant un cahier des charges détaillant le résultat attendu, notamment : la possibilité d'afficher le texte reconstitué de chaque manuscrit ; l'affichage de diverses informations encodées comme la distinction des mains, les ajouts ou suppression, l'apparat critique ; l'ouverture de chaque glose dans une infobulle à partir d'un clic sur le lemme dans le texte central. Un prototype de site de consultation a donc été créé à partir de fichiers HTML obtenus par transformation XSLT des fichiers encodés en XML-TEI sur un échantillon de texte constitué par le prologue du De plantis ${ }^{51}$. Les fonctionnalités de ce prototype, fondées sur le langage JavaScript, doivent encore être affinées en vue de la publication du site.

\section{Création d'un environnement de balisage dédié}

En ce qui concerne l'encodage du fichier, on a écarté certaines solutions qui ont pu être retenues par les projets évoqués ci-dessus :

- partir d'un fichier Word ou OpenOffice stylé et obtenir un fichier XML-TEI via une transformation XSLT

Cette solution nécessite de limiter la complexité de l'encodage, à moins de la considérer comme un prébalisage utile lorsque l'on dispose déjà d'une transcription dans un fichier texte, prébalisage qui peut être complété par un encodage plus fin une fois le fichier XML-TEI obtenu. 
- utiliser CollateX, programme de collation automatique de transcriptions, capable de produire un fichier XML-TEI encodé selon la méthode " parallel segmentation " déjà évoquée

L'utilisation de ce programme est plutôt recommandée pour les textes assez cohérents, dont on a déjà une numérisation (OCR ou transcription), et qui ne présentent pas de variantes importantes. De plus, de mon point de vue, la phase d'encodage manuel des variantes permet de réaliser une préanalyse philologique.

Ensuite, un environnement de balisage disposant de commandes adaptées à la structure de la glose a été créé afin de construire le cadre de travail configuré pour l'encodage du texte d'Aristote, des gloses marginales et interlinéaires, des liens entre les lemmes du texte et les gloses (au moyen d'attributs pointeurs), mais aussi de l'apparat critique du texte central et des gloses et de l'identification des sources avec leur indexation. Le programme utilisé pour développer cet environnement de balisage est l'éditeur XMLMind XML Editor ${ }^{52}$, mais un framework équivalent pour l'encodage de l'apparat critique selon la méthode " parallel segmentation" a également été développé sous l'éditeur oXygen XML pour permettre de traiter les textes en scripts RTL (right to left), comme l'hébreu ou l'arabe, dans le cadre d'un projet de la section hébraïque de l'IRHT $^{53}$.

\section{Objectifs plus larges, prolongements et collaboration}

L'objectif plus large est en effet la constitution d'un centre de ressources et de soutien pour les chercheurs du laboratoire impliqués dans des entreprises d'édition de sources. Les outils proposés (environnements d'encodage en XML-TEI configurables, feuilles de transformation, méthodologies d'encodage) pourront être configurés pour chaque projet et permettront aux chercheurs qui le souhaitent de réaliser plus facilement une édition scientifique électronique, tout en fournissant des données plus aisées à traiter pour les ingénieurs informaticiens, déjà engagés dans de nombreux projets et dans la maintenance et la mise à jour des projets aboutis. Lorsque ces outils auront été suffisamment testés et seront arrivés à maturité, ils pourront être proposés à un public plus large au sein d'un portail de ressources potentiellement lié aux réalisations de l'Equipex Biblissima.

Lédition électronique du texte glosé du De plantis et des Meteora formera le noyau d'un futur portail d'éditions de sources en XML-TEI à l'IRHT, pendant de Telma ${ }^{54}$, davantage tourné vers des éditions de textes diplomatiques et de bases de données.

Un séminaire mensuel sur la question des humanités numériques appliquées aux textes manuscrits (2019 et 2020, IRHT, Paris) permet d'organiser une discussion sur des questions pratiques avec des collègues français ou étrangers. La première séance du séminaire a été consacrée à la transformation depuis le XML-TEI vers LaTeX. La communication sur les avancées du projet est assurée grâce à un carnet de recherche sur la plateforme Hypothèses ${ }^{55}$ : http://digigloses.hypotheses.org. La collaboration scientifique est facilitée par l'environnement académique du projet, et notamment la Societas artistarum, fondée par Olga Weijers et Dragos Calma dans le but de promouvoir les études sur la faculté des arts, et dont certains membres ont abordé la question de la glossa Angli- 
cana dans leurs travaux ; de même avec les responsables du projet d'édition du commentaire d'Adam de Buckfield au De memoria et reminiscentia, Julie Brumberg-Chaumont et Dominique Poirel.

Sur le plan technique, les attentes portent sur une collaboration libre avec le pôle Document numérique de la MRSH de Caen, notamment dans le cadre d'une standardisation souhaitée des pratiques et d'un mouvement vers une ouverture des données, mais aussi vers leur pérennité, et vers l'adoption de méthodologies communes et d'outils communs suffisamment configurables pour permettre une adaptabilité aux spécificités des projets ${ }^{56}$.

\section{Bibliographie}

Andrée, Alexander. 2005. Gilbertus Universalis : Glossa ordinaria in Lamentationes Ieremie prophete. Prothemata et Liber I. A Critical Edition with an Introduction and a Translation. Acta Universitatis Stockholmiensis. Studia latina Stockholmiensia 52. Stockholm : Almqvist \& Wiksell International.

Andrée, Alexander. 2016. «Editing the Gloss (later Glossa ordinaria) on the Gospel of John : A Structural Approach ». Dans The Arts of Editing Medieval Greek and Latin : A Casebook, édité par Elisabet Göransson, Gunilla Iversen et Barbara Crostini, 2-21. Toronto : Pontifical Institute of Medieval Studies.

Bernhard, Michael et Calvin M. Bower, éd. 1993. Glossa maior in Institutionem musicam Boethii. Munich : Verlag der Bayerischen Akademie der Wissenschaft.

Boot, Peter et Joris van Zundert. 2011. « The Digital Edition 2.0 and The Digital Library : Services, not Resources ». Dans Digitale Edition und Forschungsbibliothek, édité par Christiane Fritze, Franz Fischer et Patrick Sahle, 141-152. Wiesbaden : Harrassowitz.

Brams, Jozef et Fernand Bossier, éd. 1990. Physica : Translatio Vetus. Leiden : Brill.

Burnett, Charles. 1996. "The Introduction of Aristotle's Natural Philosophy in Great Britain : A Preliminary Survey of the Manuscript Evidence ». Dans Aristotle in Britain during the Middle Ages, édité par John Marenbon, 21-50. Turnhout : Brepols.

Burnett, Charles et Andrew Mendelsohn. 1997. " Aristotle and Averroes on Method in the Middle Ages and Renaissance : the 'Oxford Gloss' to the Physics and Pietro d'Afeltro's $E x$ positio Proemii Averroys ». Dans Method and Order in Renaissance Philosophy of Nature. The Aristotle Commentary Tradition, édité par Daniel A. Di Liscia, Eckhard Kessler et Charlotte Methuen, 53-111. Aldershot: Ashgate.

Donati, Silvia. 1998. «Il commento alla Fisica di Adamo di Bocfeld e un commento anonimo della sua scuola ». Documenti e studi sulla tradizione filosofica medievale 9 et $10: 111-178$ et 233-298.

Dove, Mary, éd. 1997. Glossa ordinaria in Canticum canticorum. Turnhout : Brepols.

Drossaart Lulofs, Hendrik J. et Evert L.J. Poortman, éd. 1989. Nicolaus Damascenus, De Plantis. Five Translations. Amsterdam : North-Holland Publishing Company.

French, Edmund J. 1998. " Adam of Buckfield and the Early Universities ». University of London.

French, Roger K. 1997a. « Teaching Aristotle in the Medieval English Universities : De plantis and the Physical Glossa ordinaria ". Physis. Rivista internazionale di storia della scienza 34 : 225-296.

French, Roger K. 1997b. «The Use of Alfred of Shareshill's Commentary on De Plantis in University Teaching in the Thirteenth-century». Viator $28: 223-251$.

French, Roger K. 2000. "Where the Philosopher Finishes, the Physician Begins : Medicine and the Arts Course in Thirteenth-century Oxford ». Dynamis 20 : 75-106.

Galle, Griet 2008. "Edition and Discussion of the Oxford Gloss on De sensu I ». Archives d'histoire doctrinale et littéraire du Moyen Âge 75 : 197-281.

Grazzini, Stefano, éd. 2011. Scholia in Iuvenalem recentiora. Pisa : Edizioni della Normale. 
Kuhry, Emmanuelle. 2014. « La Compilatio de libris naturalibus Aristotelis et aliorum quorundam philosophorum ou Compendium philosophie : histoire et édition préliminaire partielle d'une compilation philosophique du XIII ${ }^{\mathrm{e}}$ siècle ". Thèse de doctorat, université de Lorraine.

Kuhry, Emmanuelle. 2019. "The Compilatio de Libris Naturalibus Aristotelis (Compendium Philosophie) : Evidence for the Early Reception of the Glossa Anglicana and of Adam of Buckfield's Commentaries ». Recherches de théologie et de philosophie médiévales.

Lacombe, Georges, Etzio Franceschini, Marthe Dulong et Aleksander Birkenmajer. 1939. Aristoteles Latinus. Pars prior. Descripsit codices. Union académique internationale. Corpus philosophorum Medii Aevi. Roma : Libreria dello Stato.

Lacombe, Georges, Lorenzo Minio Paluello, Etzio Franceschini, Marthe Dulong et Aleksander Birkenmajer. 1955. Aristoteles Latinus. Pars posterior. Codices descripsit. Union académique internationale. Corpus philosophorum Medii Aevi. Cambridge : University Press.

Lafleur, Claude et Joanne Carrier, éd. 1997. L'Enseignement de la philosophie au XIII ${ }^{e}$ siècle : autour du "Guide de létudiant " du ms. Ripoll 109. Studia Artistarum 5. Turnhout : Brepols.

Long, Raymond J. 1985. « Alfred of Sareshel's Commentary on the Pseudo-Aristotelian De plantis : A Critical Edition ». Mediaeval Studies 47 : 125-167.

Long, Raymond J., éd. 2013. Adam of Bockenfield, Glossae super De vegetabilibus et plantis : A Critical Edition with Introduction. Leiden : Brill.

Migne, Jacques-Paul, éd. 1852. Walafridi Strabi Fuldensis monachi opera omnia 1. Vol. 113. Patrologiae cursus completus. Series Latina. Paris : Garnier.

Minio Paluello, Lorenzo. 1961. Aristoteles Latinus Codices. Supplementa altera. Union académique internationale. Corpus philosophorum Medii Aevi. Bruges : Desclée de Brouwer.

Orbán, Arpád Peter, éd. 2006. Aratoris Subdiaconi Historia apostolica. 2 vol. Turnhout : Brepols.

O'Sullivan, Sinéad, éd. 2004. Early Medieval Glosses On Prudentius' Psychomachia : The Weitz Tradition. Leiden : Brill.

O’Sullivan, Sinéad. 2010. Glossae aevi Carolini in libros I-II Martiani Capellae De nuptiis Philologiae et Mercurii. Vol. 237. Corpus Christianorum. Continuatio mediaevalis. Turnhout : Brepols.

Rubino, Elisa, éd. 2010. Aristoteles Latinus 10.1. Meteorologica : liber quartus, transl. Henrici Aristippi. Corpus philosophorum Medii Aevi. Turnhout : Brepols.

San Juan Manso, Enara, éd. 2015. El Commentum Monacense a Terencio. Vitoria : Universidad del País Vasco.

Schoonheim, Pieter L., éd. 20oo. Aristotle's Meteorology in the Arabico-Latin Tradition : A Critical Edition of the Texts, with Introduction and Indices. Leiden : Brill.

Teeuwen, Mariken. 2007. « The Impossible Task of Editing a Ninth-century Commentary : the Case of Martianus Capella ». Variants $6: 191-208$.

Teeuwen, Mariken. 2011. « Marginal Scholarship : Rethinking the Function of Latin Glosses in Early Medieval Manuscripts ». Dans Rethinking and Recontextualizing Glosses, édité par Patrizia Lendinara, Loredana Lazzari et Claudia Di Sciacca, 19-38. Turnhout : Brepols.

Teeuwen, Mariken. 2012. "The Digital Edition : New Possibilities and Challenges». Apocrypha. Revue internationale des littératures apocryphes 23:155-168.

Teeuwen, Mariken. 2014. «"The Master has it wrong”. Dissenting Voices in Commentary Texts ». Dans Auctor et auctoritas in Latinis Medii Aevi litteris, édité par Edoardo D’Angelo et Jan M. Ziolkowski, 1097-1108. Firenze : SISMEL et Edizioni del Galluzzo.

Teeuwen, Mariken, Mary Garrison, Sinead O'Sullivan, Natalia Lozovsky, Bruce S. Eastwood et Jean-Yves Guillaumin. 2008. Carolingian Scholarship and Martianus Capella : The Oldest Commentary Tradition. Digital edition. http://martianus.huygens.knaw.nl.

Thomson, Rodney M. 2011. Catalogue of Medieval Manuscripts of Latin Commentaries on Aristotle in British Libraries. 1, Oxford. Turnhout : Brepols.

Thomson, Rodney M. 2013. Catalogue of Medieval Manuscripts of Latin Commentaries on Aristotle in British Libraries. 2, Cambridge. Turnhout : Brepols.

Ventura, Iolanda, éd. 2007. Barthélemy l'Anglais, De proprietatibus rerum, vol. VI : Liber XVII. De diversis artibus 79. Turnhout : Brepols. 
Weijers, Olga. 1988. «L'appellation des disciplines dans les classifications des sciences aux $\mathrm{XI}^{\mathrm{e}}$ et XIII ${ }^{\mathrm{e}}$ siècles ". Archivum Latinitatis Medii Aevi 46-47 : 39-64.

Weijers, Olga et Louis Holtz, éd. 1997. L'Enseignement des disciplines à la faculté des arts (Paris et Oxford, XIII ${ }^{e}-\mathrm{XV}^{e}$ S.). Actes du colloque international, 18-20 mai 1995. Studia Artistarum 4. Turnhout: Brepols.

Weisheipl, James A. 1965. «Classification of the Sciences in Medieval Thought ». Medieval Studies 27 : 54-90.

Wieland, Gernot R. 1998. "Interpreting the Interpretation : The Polysemy of the Latin Gloss ». The Journal of Medieval Latin 8 : 59-71.

Williams, Steven J. 1995. « Defining the Corpus Aristotelicum : Scholastic Awareness of Aristotelian Spuria in the High Middle Ages ». Journal of the Warburg and Courtauld Institutes $58: 25-51$.

Zetzel, James E.G. 2005. Marginal Scholarship and Textual Deviance. The Commentum Cornuti and the Early Scholia on Persius. London : Institute of Classical Studies, School of Advanced Study, University of London.

Zier, Mark A. 1993. "The Manuscript Tradition of the Glossa ordinaria for Daniel, and Hints at a Method for a Critical Edition». Scriptorium 47 : 3-25.

\section{Annexe}

Liste des manuscrits potentiels de la glossa Anglicana repérés

1. Cambridge, Gonville et Caius College 452/379

2. Cambridge, Gonville et Caius College 506/384

3. Cambridge, University Library Ee.II.31

4. Vatican, BAV Urb. lat. 206

5. Vatican, BAV Vat. lat. 2071

6. Durham, Chapter Lib. CIII 17

7. Durham, Chapter Lib. CIV 18

8. Escorial, Biblioteca real f.II.4

9. Erfurt, Universitätsbibliothek Amplon. Fol. 29

10. Florence, Biblioteca Medicea Laurenziana Plut. 13 Sin. 5

11. Florence, Biblioteca Medicea Laurenziana Plut. 14 Sin.1

12. Florence, Biblioteca Nazionale Centrale, Conv. Soppr. I.IV.22

13. Cracovie, Biblioteka Jagiellońska 689

14. Cracovie, Biblioteka Jagiellońska 758

15. Londres, British Library Royal 12.G.2

16. Londres, British Library Royal 12.G.3

17. Londres, British Library Royal 12.G.5

18. Londres, British Library Harley 3487

19. Milan, Biblioteca Ambrosiana C 148 Inf.

20. Madrid, Biblioteca nacional 1428

21. Madrid, Biblioteca nacional 9726

22. Nuremberg, Stadtbibliothek Cent V 59

23. Oxford, Corpus Christi College 111 


\author{
24. Oxford, Corpus Christi College 114 \\ 25. Paris, BNF lat. 12953 \\ 26. Schlägl, Stiftsbibliothek $10 \mathrm{Cpl}[456 \mathrm{~b}] 20$
}

\title{
Notes
}

1 Je voudrais particulièrement remercier F. Duplessis et J.-P. Boudet pour leur relecture.

2 Ce corpus vetustius est constitué de traductions arabo-latines et gréco-latines des textes aristotéliciens sur la nature réalisées jusque dans la première moitié du XIII ${ }^{\mathrm{e}}$ siècle, après quoi il est progressivement remplacé par les nouvelles traductions gréco-latines de Guillaume de Moerbecke.

3 La " chaîne de gloses scolaires " constituée à Oxford est identifiée premièrement par F. Bossier et J. Brams dans leur édition de la translatio vetus de la Physica (Brams et Bossier 1990, LV).

4 Voir Drossaart Lulofs et Poortman (1989). Également appelé De vegetabilibus ou De vegetabilibus et plantis par les auteurs qui le citent, le texte, traduit de l'arabe par Alfred de Sharshill à la fin du XII ${ }^{\mathrm{e}}$ siècle, passe jusqu'au Xvi ${ }^{\mathrm{e}}$ siècle pour un écrit authentiquement aristotélicien, bien que des doutes sur son authenticité soient émis dès le dernier quart du XIII $^{\mathrm{e}}$ siècle (Williams 1995, 43).

5 Voir Pieter L. Schoonheim (2000) et Rubino (2010). Le traité météorologique d'Aristote circule à partir du XIII ${ }^{\mathrm{e}}$ siècle dans la traduction arabo-latine de Gérard de Crémone pour les livres I-III, gréco-latine d'Henri Aristippe pour le livre IV et d'Alfred de Shareshill pour l'appendice minéralogique au livre IV De mineralibus provenant du Kitāb al-shif $\bar{a}^{\text {' }}$ d'Avicenne.

6 https://tei-c.org.

7 On note l'absence, dans les textbooks, du De animalibus, le traité zoologique d'Aristote, qui, dans la traduction arabo-latine de Michel Scot, comprenait l'Histoire, les Parties et la Génération des animaux. Ce texte circulait généralement à part.

8 Voir la section : liste des éditions.

9 Voir la liste des manuscrits donnée en annexe.

10 D'autres témoins de la glose conservés sur le continent sont à prendre en compte : par exemple, le $\mathrm{n}^{0} 25$ de la liste comporte le De plantis glosé.

11 Sur les classifications des sciences en usage au Moyen Âge, on pourra lire aussi Weisheipl (1965) et Weijers (1988).

12 Thèse soutenue en 2014 à l'université de Lorraine sous la direction d'I. Draelants, directrice de recherche au CNRS, et de C. Jacquemard, professeur à l'université de Caen, voir Kuhry (2014; 2019).

13 Roger K. French indique que le contenu avicennien peut aussi se cacher sous la référence à un "commentator» (French 2000, 86). Le Canon (al-Qānün fì aț-Tỉbb) d'Avicenne fut traduit en latin par Gérard de Crémone dans la seconde moitié du XII ${ }^{\mathrm{e}}$ siècle. Le terme «commentator» utilisé dans la glose désigne aussi généralement Averroès.

14 Londres, British Library Harley 3487, f. 158v : «[demonis] : id est arcum scientis, unde Commentator super librum eticorum dicit quod demon aliquando significat intelligentiam, aliquando stellam preclaram, [...] demonis id est arcus angelice considerationis, tamquam ad eius notitiam humanus non ascendit intellectus, unde dicit quod demon sonat idem quod angelus. Item dicit quod secundum Salomom israelitam arcus iste dicitur arabice cascuza quod interpretatur arcus multicolor sive varius. » Cf. Albertus Magnus, Meteora, III, IV.

15 Peu des commentaires d'Adam de Buckfield sont édités. Le commentaire au De plantis est édité par R. J. Long, ce qui facilitera le repérage de parallèles avec la glose d'Oxford sur le De plantis (Long 2013). Une édition du commentaire d'Adam de Buckfield au De memoria et reminiscentia d'Aristote est en cours de publication par D. Poirel et J. BrumbergChaumont.

16 Gernot R. Wieland utilise le terme de " commentaire " pour toute collection de gloses sans distinction formelle, tandis que J. E. G. Zetzel distingue bien le commentaire (livré séparément du texte intégral) de la scholie, tout en précisant qu'il arrive souvent que la 
limite entre les deux soit floue (Wieland 1998, 59-60) ; (Zetzel 2005, 86). M. Teeuwen discute la pertinence de cette distinction (Teeuwen 2007, 197-199).

17 Dans un article en voie de publication (Kuhry 2019), je donne l'exemple d'un malentendu au sujet du nom des vents dans la glose d'Oxford sur les Meteora, qu'une partie de la tradition manuscrite interprète de manière erronée tout en mentionnant l'existence de l'opinion contraire. Sur ce sujet on peut lire aussi Teeuwen (2014).

18 En ce qui concerne les gloses du haut Moyen Âge, M. Teeuwen insiste sur le caractère savant de l'acte de gloser plutôt qu'uniquement didactique (Teeuwen 2011, 23-24). En particulier, les gloses jettent des ponts vers d'autres textes et créent ainsi un tissu intertextuel.

19 Au sujet du lien entre le De mineralibus et les Meteora, voir n. 5 .

20 Voir la section «Liste des éditions ».

21 http://v-machine.org; http://ciham-digital.huma-num.fr/teitoolbox/witnesses.php.

22 Citons notamment le projet néerlandais Marginal Scholarship. The Practice of Learning in the Early Middle Ages (c. 80o - c. 100o) (2011-2015) mené par M. Teeuwen (Huygens Instituut voor Nederlandse Geschiedenis, voir notamment Teeuwen et al. [2008]) et le projet d'édition électronique de la glose ordinaire de la Bible mené par M. Morard (IRHT-CNRS) depuis 2006 (http://gloss-e.irht.cnrs.fr).

23 Dans certains manuscrits de la traduction vetus des Meteora, comme les $\mathrm{n}^{\text {os }} 4$ et 18 de la liste, au sujet de l'arc-en-ciel (livre 3, chap. 7), le terme demonis est apposé à l'expression arcus varius. Les autres manuscrits du corpus vetustius portant la glossa Anglicana ne comportent pas cet ajout dans le texte d'Aristote. Néanmoins, la glose sur le lemme demonis est présente dans tous les manuscrits de la glossa, même lorsque le lemme est absent du texte.

$24 \mathrm{http} / / / w w w . l a t e x-p r o j e c t . o r g$.

25 Les différents packages de LaTeX utilisables dans le contexte d'une édition critique sont passés en revue sur le carnet de recherche de J.-B. Camps : http://graal.hypotheses.org/484. Citons en particulier les packages Reledmac et Reledpar, maintenus par M. Rouquette.

26 Quatre volumes disponibles en ligne :

https://archive.thulb.uni-jena.de/ufb/receive/ufb_cbu_00000254, https://archive.thulb.uni-jena.de/ufb/receive/ufb_cbu_00000255, https://archive.thulb.uni-jena.de/ufb/receive/ufb_cbu_00000256, https://archive.thulb.uni-jena.de/ufb/receive/ufb_cbu_00000227.

Cette édition est la base du projet d'édition électronique de la glose biblique par M. Morard (IRHT) : http://gloss-e.irht.cnrs.fr.

27 http://orosius.monumenta.ch.

28 http://mlat.uzh.ch/MLS/.

29 https://www.e-codices.unifr.ch/fr/.

$30 \mathrm{http}: / /$ martianus.huygens.knaw.nl. La méthodologie adoptée et l'outil utilisé sont décrits dans Teeuwen (2012). L'outil e-laborate propose un environnement d'édition en ligne et collaboratif qui permet de publier directement l'édition électronique obtenue : voir http ://elaborate.huygens.knaw.nl.

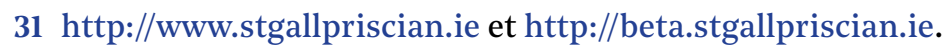

32 http://gloss-e.irht.cnrs.fr.

$33 \mathrm{http}: / /$ www.martianus.mueze.lmu.de.

34 http://www.persius.mueze.lmu.de/ghtm/gfr.htm.

$35 \mathrm{http}: / /$ www.persius.mueze.lmu.de/persius_code.pdf.

36 http://liber-glossarum.huma-num.fr.

37 http://liber-glossarum.huma-num.fr/the_Libgloss_project_fr.html et http://liber-glos sarum.huma-num.fr/encodage.html.

38 http://www.tei-c.org/release/doc/tei-p5-doc/en/html/TC.html\#TCAPLO.

39 http://www.huma-num.fr.

40 M. Teeuwen formulait un vœu allant dans ce sens (Teeuwen 2007, 203-204). Voir aussi Teeuwen $(2012,163)$. 
41 http://elaborate.huygens.knaw.nl/?page_id=105 : "In some cases, eLaborate is not the best tool for the creation of a text edition. For instance, eLaborate is not suited if one wants to make a aligned synoptic text edition (but if alignment is not needed, eLaborate can be a serious option). When a text or text collection is very complex as to the genetic background and would need very complex layers of annotations, Huygens ING advises the use of XML. "

42 C'est l'une des idées qui ressort du livre blanc du CNRS, Une science ouverte dans une République numérique : $\quad \mathrm{http} / / / \mathrm{www} . \mathrm{cnrs.fr} / \mathrm{dist} / \mathrm{z}$-outils/documents/2016\%2003\%2024\%20Livre\%20blanc\%200pen\%20Science.pdf.

$43 \mathrm{http} / / /$ textometrie.ens-lyon.fr.

44 http://www.tei-c.org/release/doc/tei-p5-doc/en/html/TC.html\#TCAPPS.

45 Cf. n. 21.

46 https://www.unicaen.fr/puc/sources/malaterra/accueil.

47 http://www.bvh.univ-tours.fr.

48 http://teicat.huma-num.fr/print.php.

$49 \mathrm{http} / /$ www.unicaen.fr/recherche/mrsh/document_numerique/. Voir les réalisations du pôle Document numérique de la MRSH de Caen : http://www.unicaen.$\mathrm{fr} / \mathrm{puc} / \mathrm{sources} / \mathrm{malaterra} / \mathrm{tdm}$ et https://www.unicaen.fr/puc/sources/depiscibus/tdm.

50 http://outils.biblissima.fr/fr/outils-edition-xml/.

51 La réalisation de ce prototype a été rendue possible grâce à un stage de première année obtenu par l'IRHT et effectué du 2 au 31 juillet 2018 par A. Plateau (Polytech Orléans) sous la direction de C. Masset.

52 Voir les utilisations de cet outil par le pôle Document numérique de la MRSH de Caen : http://www.unicaen.fr/recherche/mrsh/document_numerique/outils/.

53 Projet Racines porté par J. Kogel.

$54 \mathrm{http} / / / \mathrm{www} . \mathrm{cn}$-telma.fr.

55 http://fr.hypotheses.org.

56 Ces aspects correspondent aux vœux formulés depuis longtemps par la communauté, avec un accent sur l'indispensable pérennité des données électroniques et la nécessité de développer moins de solutions d'affichage ad hoc et plus d'outils communs, voir notamment Boot et Zundert (2011).

\section{Auteur}

\section{Emmanuelle Kuhry}

UPR 841 IRHT, CNRS, Orléans, France

Emmanuelle Kuhry est post-doctorante à l'Institut de recherche et d'histoire des textes. Ses travaux portent sur l'histoire des textes scientifiques médiévaux, en particulier des textes de philosophie naturelle. Dans sa thèse, elle a étudié une compilation aristotélicienne anonyme du milieu du xIII ${ }^{\mathrm{e}}$ siècle, dont elle a réalisé l'édition critique d'une partie du texte au moyen du XML-TEI et de LaTeX.

emmanuelle.kuhry@irht.cnrs.fr

\section{Droits d'auteur}

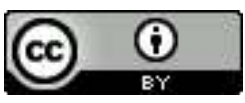

Les contenus de la revue Humanités numériques sont mis à disposition selon les termes de la Licence Creative Commons Attribution 4.0 International. 